\title{
Nonnegative Solutions for a Riemann-Liouville Fractional Boundary Value Problem
}

\author{
Rodica Luca1 ${ }^{1}$, Alexandru Tudorache ${ }^{2}$ \\ ${ }^{1}$ Department of Mathematics, Gh. Asachi Technical University, Iasi, Romania \\ ${ }^{2}$ Faculty of Computer Engineering and Automatic Control, Gh. Asachi Technical University, Iasi, Romania \\ Email: rluca@ma th.tuia si.ro, alexandru.tudorache93@gmail.co
}

How to cite this paper: Luca, R. and Tudorache, A. (2019) Nonnegative Solutions for a Riemann-Liouville Fractional Boundary Value Problem. Open Journal of Applied Sciences, 9, 749-760.

https://doi.org/10.4236/ojapps.2019.910061

Received: September 13, 2019

Accepted: October 14, 2019

Published: October 17, 2019

Copyright $\odot 2019$ by author(s) and Scientific Research Publishing Inc. This work is licensed under the Creative Commons Attribution International License (CC BY 4.0).

http://creativecommons.org/licenses/by/4.0/

\begin{abstract}
We investigate the existence of nonnegative solutions for a Riemann-Liouville fractional differential equation with integral terms, subject to boundary conditions which contain fractional derivatives and Riemann-Stieltjes integrals. In the proof of the main results, we use the Banach contraction mapping principle and the Krasnosel'skii fixed point theorem for the sum of two operators.
\end{abstract}

\section{Keywords}

Riemann-Liouville Fractional Differential Equations, Nonlocal Boundary Conditions, Nonnegative Solutions, Existence

\section{Introduction}

We consider the nonlinear fractional differential equation

$$
D_{0+}^{\alpha} x(t)+f(t, x(t), A x(t), B x(t))=0, t \in(0,1),
$$

with the nonlocal boundary conditions

$$
x(0)=x^{\prime}(0)=\cdots=x^{(n-2)}(0)=0, D_{0+}^{\beta_{0}} x(1)=\sum_{i=1}^{m} \int_{0}^{1} D_{0+}^{\beta_{i}} x(t) \mathrm{d} H_{i}(t),
$$

where $\alpha \in \mathbb{R}, \alpha \in(n-1, n], n, m \in \mathbb{N}, n \geq 3, \quad \beta_{i} \in \mathbb{R}$ for all $i=0, \cdots, m$, $0 \leq \beta_{1}<\beta_{2}<\cdots<\beta_{m} \leq \beta_{0}<\alpha-1, \quad \beta_{0} \geq 1, D_{0+}^{k}$ denotes the Riemann-Liouville derivative of order $k$ (for $k=\alpha, \beta_{0}, \beta_{1}, \cdots, \beta_{m}$ ), the integrals from the boundary condition $(B C)$ are Riemann-Stieltjes integrals with $H_{i}:[0,1] \rightarrow \mathbb{R}, i=1, \cdots, m$ non-decreasing functions, $A x(t)=\int_{0}^{t} K(t, s) x(s) \mathrm{d} s$, and $B x(t)=\int_{0}^{1} H(t, s) x(s) \mathrm{d} s$ for all $t \in[0,1]$.

We study the existence of nonnegative solutions for problem $(E)-(B C)$ by using the Banach contraction mapping principle and the Krasnosel'skii fixed point 
theorem for the sum of two operators. Equation $(E)$ supplemented with the multi-point boundary conditions

$$
x(0)=x^{\prime}(0)=\cdots=x^{(n-2)}(0)=0, D_{0+}^{p} x(1)=\sum_{i=1}^{m} a_{i} D_{0+}^{q} x\left(\xi_{i}\right),
$$

where $a_{i}, \xi_{i} \in \mathbb{R}$ for all $i=1, \cdots, m,(m \in \mathbb{N}), 0<\xi_{1}<\cdots<\xi_{m} \leq 1, \quad p, q \in \mathbb{R}$, $p \in[1, n-2], q \in[0, p]$ was investigated in the paper [1]. The last condition in $\left(B C_{1}\right)$ can be written as $D_{0+}^{p} x(1)=\int_{0}^{1} D_{0+}^{q} x(t) \mathrm{d} H_{0}(t)$, where $H_{0}$ is the step function defined by

$$
H_{0}(t)=\left\{0, \text { for } t \in\left[0, \xi_{1}\right) ; a_{1} \text {, for } t \in\left[\xi_{1}, \xi_{2}\right) ; a_{1}+a_{2}, \text { for } t \in\left[\xi_{2}, \xi_{3}\right) ; \cdots ; \sum_{i=1}^{m} a_{i} \text {, for } t \in\left[\xi_{m}, 1\right]\right\}
$$

So $(B C)$ is a generalization of $\left(B C_{1}\right)$, because in $(B C)$ we have a sum of Riemann-Stieltjes integrals and various orders for the fractional derivatives. In the paper [2], the authors investigated the existence of nonnegative solutions for the Caputo fractional differential equation

$$
{ }^{c} D^{\alpha} x(t)+f(t, x(t), A x(t), B x(t))=0, t \in(0,1),
$$

with the boundary conditions

$$
\left(B C_{2}\right) x(0)=b_{0}, x^{\prime}(0)=b_{1}, \cdots, x^{(n-3)}(0)=b_{n-3}, x^{(n-1)}(0)=b_{n-1}, x(1)=\mu \int_{0}^{1} x(s) \mathrm{d} s,
$$

where $n-1<\alpha \leq n, 0 \leq \mu<n-1, \quad n \geq 3, \quad b_{i} \geq 0 \quad(i=1,2, \cdots, n-3, n-1),{ }^{c} D^{\alpha}$ is the Caputo fractional derivative, and the operators $A$ and $B$ are defined as the operators from our problem, given above. In the paper [3], the authors studied the existence and multiplicity of positive solutions for the Riemann-Liouville fractional differential equation $D_{0+}^{\alpha} x(t)+f(t, x(t))=0, t \in(0,1)$, subject to the boundary conditions $(B C)$, where $f$ is a sign-changing function that can be singular in the points $t=0,1$ and/or in the variable $x$. In addition, the methods used in the proofs of the main results in [3] are different than those used in the present paper, namely, in [3] the authors used various conditions which contain height functions of the nonlinearity defined on special bounded sets, and two theorems from the fixed point index theory. For some recent results on the existence, nonexistence and multiplicity of solutions for fractional differential equations and systems of fractional differential equations subject to various boundary conditions we refer the reader to the monographs [4] [5] and the papers [6]-[14]. We also mention the books [15]-[21], and the papers [22]-[28] for applications of the fractional differential equations in various disciplines.

\section{Preliminary Results}

We present in this section some auxiliary results from [3] that we will use in the proof of the main results. We consider the fractional differential equation

$$
D_{0+}^{\alpha} x(t)+y(t)=0, t \in(0,1),
$$

with the boundary conditions $(B C)$, where $y \in C(0,1) \cap L^{1}(0,1)$. We denote by 
$\Delta=\frac{\Gamma(\alpha)}{\Gamma\left(\alpha-\beta_{0}\right)}-\sum_{i=1}^{m} \frac{\Gamma(\alpha)}{\Gamma\left(\alpha-\beta_{i}\right)} \int_{0}^{1} s^{\alpha-\beta_{i}-1} \mathrm{~d} H_{i}(s)$.

Lemma 1 If $\Delta \neq 0$, then the unique solution $x \in C[0,1]$ of problem (1)- $(B C)$ is given by

$$
\begin{aligned}
x(t)= & -\frac{1}{\Gamma(\alpha)} \int_{0}^{t}(t-s)^{\alpha-1} y(s) \mathrm{d} s+\frac{t^{\alpha-1}}{\Delta \Gamma\left(\alpha-\beta_{0}\right)} \int_{0}^{1}(1-s)^{\alpha-\beta_{0}-1} y(s) \mathrm{d} s \\
& -\frac{t^{\alpha-1}}{\Delta} \sum_{i=1}^{m} \frac{1}{\Gamma\left(\alpha-\beta_{i}\right)} \int_{0}^{1}\left(\int_{0}^{s}(s-\tau)^{\alpha-\beta_{i}-1} y(\tau) \mathrm{d} \tau\right) \mathrm{d} H_{i}(s), t \in[0,1] .
\end{aligned}
$$

Lemma 2 If $\Delta \neq 0$, then the solution $x$ of problem (1)-(BC) given by (2) can be written as

$$
x(t)=\int_{0}^{1} G(t, s) y(s) \mathrm{d} s, t \in[0,1],
$$

where

$$
G(t, s)=g_{1}(t, s)+\frac{t^{\alpha-1}}{\Delta} \sum_{i=1}^{m}\left(\int_{0}^{1} g_{2 i}(\tau, s) \mathrm{d} H_{i}(\tau)\right)
$$

and

$$
\begin{aligned}
& g_{1}(t, s)=\frac{1}{\Gamma(\alpha)}\left\{\begin{array}{l}
t^{\alpha-1}(1-s)^{\alpha-\beta_{0}-1}-(t-s)^{\alpha-1}, 0 \leq s \leq t \leq 1, \\
t^{\alpha-1}(1-s)^{\alpha-\beta_{0}-1}, 0 \leq t \leq s \leq 1,
\end{array}\right. \\
& g_{2 i}(t, s)=\frac{1}{\Gamma\left(\alpha-\beta_{i}\right)}\left\{\begin{array}{l}
t^{\alpha-\beta_{i}-1}(1-s)^{\alpha-\beta_{0}-1}-(t-s)^{\alpha-\beta_{i}-1}, 0 \leq s \leq t \leq 1, \\
t^{\alpha-\beta_{i}-1}(1-s)^{\alpha-\beta_{0}-1}, 0 \leq t \leq s \leq 1,
\end{array}\right.
\end{aligned}
$$

for all $(t, s) \in[0,1] \times[0,1], i=1, \cdots, m$.

By using some properties of the functions $g_{1}, g_{2 i}, i=1, \cdots, m$ given by (5) from [29], we obtain the following lemma.

Lemma 3 We suppose that $\Delta>0$. Then the function $G$ given by (4) is a continuous function on $[0,1] \times[0,1]$ and satisfies the inequalities.

a) $G(t, s) \leq J(s)$ for all $t, s \in[0,1]$, where

$J(s)=h_{1}(s)+\frac{1}{\Delta} \sum_{i=1}^{m} \int_{0}^{1} g_{2 i}(\tau, s) \mathrm{d} H_{i}(\tau), \quad s \in[0,1]$, and

$h_{1}(s)=\frac{1}{\Gamma(\alpha)}(1-s)^{\alpha-\beta_{0}-1}\left(1-(1-s)^{\beta_{0}}\right), \quad s \in[0,1]$;

b) $G(t, s) \geq t^{\alpha-1} J(s)$ for all $t, s \in[0,1]$;

c) $G(t, s) \leq \sigma t^{\alpha-1}$, for all $t, s \in[0,1]$, where

$\sigma=\frac{1}{\Gamma(\alpha)}+\frac{1}{\Delta} \sum_{i=1}^{m} \frac{1}{\Gamma\left(\alpha-\beta_{i}\right)} \int_{0}^{1} \tau^{\alpha-\beta_{i}-1} \mathrm{~d} H_{i}(\tau)$.

Lemma 4 We suppose that $\Delta>0, y \in C(0,1) \cap L^{1}(0,1)$ and $y(t) \geq 0$ for all $t \in(0,1)$. Then the solution $x$ of problem (1)-(BC) given by (3) satisfies the inequality $x(t) \geq t^{\alpha-1}\|x\|$ for all $t \in[0,1]$, where $\|x\|=\sup _{t \in[0,1]}|x(t)|$, and so $x(t) \geq 0$ for all $t \in[0,1]$.

In the proof of our main theorems, we use the Banach contraction mapping principle and the Krasnosel'skii fixed point theorem for the sum of two opera- 
tors presented below.

Theorem 1 (see [30]) If $(Y, d)$ is a nonempty complete metric space with the metric $d$, and $T: Y \rightarrow Y$ is a contraction mapping, then $T$ has a unique fixed point $x^{*} \in Y \quad\left(T x^{*}=x^{*}\right)$.

Theorem 2 ([31]) Let $M$ be a closed, convex, bounded and nonempty subset of a Banach space $X$. Let $A_{1}$ and $A_{2}$ be two operators such that

a) $A_{1} x+A_{2} y \in M$ for all $x, y \in M$;

b) $A_{1}$ is a completely continuous operator (continuous, and compact, that is, it maps bounded sets into relatively compact sets);

c) $A_{2}$ is a contraction mapping.

Then there exists $z \in M$ such that $z=A_{1} z+A_{2} z$.

\section{Main Results}

In this section we study the existence of nonnegative solutions for our problem (E)-(BC). We present now the assumptions that we will use in the sequel.

(II) $\alpha \in \mathbb{R}, \quad \alpha \in(n-1, n], \quad n, m \in \mathbb{N}, \quad n \geq 3, \quad \beta_{i} \in \mathbb{R}$ for all $i=0, \cdots, m$, $0 \leq \beta_{1}<\beta_{2}<\cdots<\beta_{m} \leq \beta_{0}<\alpha-1, \quad \beta_{0} \geq 1, \quad H_{i}:[0,1] \rightarrow \mathbb{R}, i=1, \cdots, m$ are nondecreasing functions, an $\Delta=\frac{\Gamma(\alpha)}{\Gamma\left(\alpha-\beta_{0}\right)}-\sum_{i=1}^{m} \frac{\Gamma(\alpha)}{\Gamma\left(\alpha-\beta_{i}\right)} \int_{0}^{1} s^{\alpha-\beta_{i}-1} \mathrm{~d} H_{i}(s)>0$.

(I2) $f:[0,1] \times \mathbb{R}_{+}^{3} \rightarrow \mathbb{R}_{+}$is measurable with respect to $t$ on $[0,1]$, $\left(\mathbb{R}_{+}=[0, \infty)\right)$.

(13) There exist the functions $a, b, c \in L^{1}\left((0,1), \mathbb{R}_{+}\right)$such that

$$
|f(t, x, y, z)-f(t, \bar{x}, \bar{y}, \bar{z})| \leq a(t)|x-\bar{x}|+b(t)|y-\bar{y}|+c(t)|z-\bar{z}|,
$$

a.e. $t \in(0,1)$ and for all $x, y, z, \bar{x}, \bar{y}, \bar{z} \in \mathbb{R}_{+}$.

(I4) There exists the function $g \in L^{1}\left((0,1), \mathbb{R}_{+}\right)$such that

$$
|f(t, x, y, z)| \leq g(t) \text {, a.e. } t \in(0,1), \forall x, y, z \in \mathbb{R}_{+} .
$$

(I5) $K \in C\left(E, \mathbb{R}_{+}\right), E=\{(t, s) \in[0,1] \times[0,1], t \geq s\}$, and $H \in C\left([0,1] \times[0,1], \mathbb{R}_{+}\right)$.

We denote by $k_{0}=\sup _{t \in[0,1]} \int_{0}^{t} K(t, s) \mathrm{d} s$ and $h_{0}=\sup _{t \in[0,1]} \int_{0}^{1} H(t, s) \mathrm{d} s$.

Theorem 3 We suppose that assumptions (I1)-(I5) hold. If $\sigma \omega_{1}<1$, where $\omega_{1}=\left\|a+k_{0} b+h_{0} c\right\|_{L^{1}}$, then problem $(E)-(B C)$ has at least one nonnegative solution on $[0,1]$.

Proof. By (I4) we obtain that the function $s \rightarrow(t-s)^{\alpha-1} f(s, x(s), A x(s), B x(s))$ is Lebesgue integrable on $[0, t]$ for all $t \in[0,1]$ and $x \in C\left([0,1], \mathbb{R}_{+}\right)$, the function

$s \rightarrow(1-s)^{\alpha-\beta_{0}-1} f(s, x(s), A x(s), B x(s))$ is Lebesgue integrable on $[0,1]$ for all $x \in C\left([0,1], \mathbb{R}_{+}\right)$, and the function $\tau \rightarrow(s-\tau)^{\alpha-\beta_{i}-1} f(\tau, x(\tau), A x(\tau), B x(\tau))$ is Lebesgue integrable on $[0, s]$ for all $s \in[0,1], \quad x \in C\left([0,1], \mathbb{R}_{+}\right)$and $i=1, \cdots, m$.

We consider now the integral equation 


$$
\begin{aligned}
& x(t)=-\frac{1}{\Gamma(\alpha)} \int_{0}^{t}(t-s)^{\alpha-1} f(s, x(s), A x(s), B x(s)) \mathrm{d} s \\
& +\frac{t^{\alpha-1}}{\Delta \Gamma\left(\alpha-\beta_{0}\right)} \int_{0}^{1}(1-s)^{\alpha-\beta_{0}-1} f(s, x(s), A x(s), B x(s)) \mathrm{d} s \\
& -\frac{t^{\alpha-1}}{\Delta} \sum_{i=1}^{m} \frac{1}{\Gamma\left(\alpha-\beta_{i}\right)} \int_{0}^{1}\left(\int_{0}^{s}(s-\tau)^{\alpha-\beta_{i}-1} f(\tau, x(\tau), A x(\tau), B x(\tau)) \mathrm{d} \tau\right) \mathrm{d} H_{i}(s),
\end{aligned}
$$

or equivalently

$$
x(t)=\int_{0}^{1} G(t, s) f(s, x(s), A x(s), B x(s)) \mathrm{d} s .
$$

By Lemma 2 we easily deduce that if $x$ is a solution of Equation (6) (or equivalently (7)), then $x$ is a solution of problem $(E)-(B C)$.

Let $r=\sigma\|g\|_{L^{L}}$. We define the operator $\mathcal{T}$ on

$$
\begin{aligned}
\bar{B}_{r} & =\left\{x \in C\left([0,1], \mathbb{R}_{+}\right),\|x\| \leq r\right\} \text { by } \\
& \mathcal{T} x(t)=-\frac{1}{\Gamma(\alpha)} \int_{0}^{t}(t-s)^{\alpha-1} f(s, x(s), A x(s), B x(s)) \mathrm{d} s \\
& +\frac{t^{\alpha-1}}{\Delta \Gamma\left(\alpha-\beta_{0}\right)} \int_{0}^{1}(1-s)^{\alpha-\beta_{0}-1} f(s, x(s), A x(s), B x(s)) \mathrm{d} s \\
& -\frac{t^{\alpha-1}}{\Delta} \sum_{i=1}^{m} \frac{1}{\Gamma\left(\alpha-\beta_{i}\right)} \int_{0}^{1}\left(\int_{0}^{s}(s-\tau)^{\alpha-\beta_{i}-1} f(\tau, x(\tau), A x(\tau), B x(\tau)) \mathrm{d} \tau\right) \mathrm{d} H_{i}(s),
\end{aligned}
$$

or equivalently

$$
\mathcal{T} x(t)=\int_{0}^{1} G(t, s) f(s, x(s), A x(s), B x(s)) \mathrm{d} s .
$$

If $x$ is a fixed point of operator $\mathcal{T}$, then $x$ is a solution of Equation (6) (or $(7)$ ), and hence $x$ is a solution of problem $(E)-(B C)$. Therefore we will study the existence (and uniqueness) of the fixed points of operator $\mathcal{T}$ by using the Banach contraction mapping principle.

We firstly show that if $x \in \bar{B}_{r}$, then $\mathcal{T} x \in \bar{B}_{r}$. Indeed, we have

$$
\begin{aligned}
& |\mathcal{T} x(t+\varepsilon)-\mathcal{T} x(t)| \\
& =\left|\int_{0}^{1}[G(t+\varepsilon, s)-G(t, s)] f(s, x(s), A x(s), B x(s)) \mathrm{d} s\right| \\
& \leq \int_{0}^{1}|G(t+\varepsilon, s)-G(t, s)| g(s) \mathrm{d} s \\
& \leq \max _{s \in[0,1]}|G(t+\varepsilon, s)-G(t, s)| \cdot\|g\|_{L^{1}} \rightarrow 0, \text { for } \varepsilon \rightarrow 0 .
\end{aligned}
$$

Hence $\mathcal{T} x$ is a continuous function. By $(I 1),(I)$ and Lemma 4, we obtain $\mathcal{T} x(t) \geq 0$ for all $t \in[0,1]$, and then $\mathcal{T} x \in C\left([0,1], \mathbb{R}_{+}\right)$.

In addition, for any $x \in \bar{B}_{r}$ and all $t \in[0,1]$, we deduce

$$
\begin{aligned}
(\mathcal{T} x)(t) & =\int_{0}^{1} G(t, s) f(s, x(s), A x(s), B x(s)) \mathrm{d} s \\
& \leq \int_{0}^{1} \sigma t^{\alpha-1} f(s, x(s), A x(s), B x(s)) \mathrm{d} s \\
& \leq \sigma t^{\alpha-1} \int_{0}^{1} g(s) \mathrm{d} s \leq \sigma\|g\|_{L^{1}}=r,
\end{aligned}
$$

and then $\|\mathcal{T} x\| \leq r$ for all $x \in \bar{B}_{r}$, so $\mathcal{T}: \bar{B}_{r} \rightarrow \bar{B}_{r}$.

We show now that $\mathcal{T}$ is a contraction mapping on $\bar{B}_{r}$. For $x_{1}, x_{2} \in \bar{B}_{r}$, and 
any $t \in[0,1]$, by using (I3), we find

$$
\begin{aligned}
& \left|\left(\mathcal{T} x_{1}\right)(t)-\left(\mathcal{T} x_{2}\right)(t)\right| \\
& \leq \int_{0}^{1} G(t, s)\left|f\left(s, x_{1}(s), A x_{1}(s), B x_{1}(s)\right)-f\left(s, x_{2}(s), A x_{2}(s), B x_{2}(s)\right)\right| \mathrm{d} s \\
& \leq \int_{0}^{1} G(t, s)\left[a(s)\left|x_{1}(s)-x_{2}(s)\right|+b(s)\left|A x_{1}(s)-A x_{2}(s)\right|\right. \\
& \left.\quad+c(s)\left|B x_{1}(s)-B x_{2}(s)\right|\right] \mathrm{d} s \\
& \leq\left\|x_{1}-x_{2}\right\| \sigma t^{\alpha-1} \int_{0}^{1}\left[a(s)+k_{0} b(s)+h_{0} c(s)\right] \mathrm{d} s \\
& \leq \sigma\left\|x_{1}-x_{2}\right\| \cdot\left\|a+k_{0} b+h_{0} c\right\|_{L^{1}}=\sigma \omega_{1}\left\|x_{1}-x_{2}\right\|,
\end{aligned}
$$

because

$$
\begin{aligned}
A x_{1}(s)-A x_{2}(s) & =\int_{0}^{s} K(s, \tau)\left(x_{1}(\tau)-x_{2}(\tau)\right) \mathrm{d} \tau \\
& \leq \sup _{\tau \in[0,1]} x_{1}(\tau)-x_{2}(\tau) \mid \int_{0}^{s} K(s, \tau) \mathrm{d} \tau \\
& \leq k_{0}\left\|x_{1}-x_{2}\right\|, \forall s \in[0,1], \\
B x_{1}(s)-B x_{2}(s) & =\int_{0}^{1} H(s, \tau)\left(x_{1}(\tau)-x_{2}(\tau)\right) \mathrm{d} \tau \\
& \leq \sup _{\tau \in[0,1]}\left|x_{1}(\tau)-x_{2}(\tau)\right| \int_{0}^{1} H(s, \tau) \mathrm{d} \tau \\
& \leq h_{0}\left\|x_{1}-x_{2}\right\|, \forall s \in[0,1],
\end{aligned}
$$

and $a, b, c \in L^{1}(0,1)$.

Therefore we obtain the inequality

$$
\left\|\mathcal{T}_{1}-\mathcal{T} x_{2}\right\| \leq \sigma \omega_{1}\left\|x_{1}-x_{2}\right\| .
$$

Because $\sigma \omega_{1}<1$, we deduce that $\mathcal{T}$ is a contraction mapping. By Theorem 1, we conclude that $\mathcal{T}$ has a unique fixed point, which is a nonnegative solution of problem $(E)-(B C)$.

In what follows, we denote by

$$
\begin{aligned}
& \omega_{2}=\frac{1}{\Gamma(\alpha)} \int_{0}^{1}(1-s)^{\alpha-1}\left(a(s)+k_{0} b(s)+h_{0} c(s)\right) \mathrm{d} s, \\
& \omega_{3}=\left\|a+k_{0} b+h_{0} c\right\|_{L^{1}}\left(\frac{1}{\Delta \Gamma\left(\alpha-\beta_{0}\right)}+\frac{1}{\Delta} \sum_{i=1}^{m} \frac{1}{\Gamma\left(\alpha-\beta_{i}\right)}\left(H_{i}(1)-H_{i}(0)\right)\right) .
\end{aligned}
$$

Theorem 4 We suppose that assumptions (I1),

$(I)^{\prime} f:[0,1] \times \mathbb{R}_{+}^{3} \rightarrow \mathbb{R}_{+}$is a continuous function and (I3), (I4), (I5) hold. If $\max \left\{\omega_{2}, \omega_{3}\right\}<1$, then problem $(E)-(B C)$ has at least one nonnegative solution on $[0,1]$.

Proof. We define $R \geq R_{0}$, where

$$
\begin{aligned}
R_{0}= & \left(1-\omega_{2}\right)^{-1}\left[\frac{\tau_{0}}{\Gamma(\alpha+1)}+\|g\|_{L^{1}}\left(\frac{1}{\Delta \Gamma\left(\alpha-\beta_{0}\right)}\right.\right. \\
& \left.\left.+\frac{1}{\Delta} \sum_{i=1}^{m} \frac{1}{\Gamma\left(\alpha-\beta_{i}\right)}\left(H_{i}(1)-H_{i}(0)\right)\right)\right],
\end{aligned}
$$

$\tau_{0}=\max \{f(t, 0,0,0), t \in[0,1]\}$ and $\omega_{2}$ is given by (8). We consider the set $\bar{B}_{R}=\left\{x \in C\left([0,1], \mathbb{R}_{+}\right),\|x\| \leq R\right\}$. Then $\bar{B}_{R}$ is a closed, convex and nonempty 
set of $C\left([0,1], \mathbb{R}_{+}\right)$. We define the operators $Q_{1}$ and $Q_{2}$ on $\bar{B}_{R}$ by $\left(Q_{1} x\right)(t)=-\frac{1}{\Gamma(\alpha)} \int_{0}^{t}(t-s)^{\alpha-1} f(s, x(s), A x(s), B x(s)) \mathrm{d} s, t \in[0,1]$, $\left(Q_{2} x\right)(t)=\frac{t^{\alpha-1}}{\Delta \Gamma\left(\alpha-\beta_{0}\right)} \int_{0}^{1}(1-s)^{\alpha-\beta_{0}-1} f(s, x(s), A x(s), B x(s)) \mathrm{d} s$ $-\frac{t^{\alpha-1}}{\Delta} \sum_{i=1}^{m} \frac{1}{\Gamma\left(\alpha-\beta_{i}\right)} \int_{0}^{1}\left(\int_{0}^{s}(s-\tau)^{\alpha-\beta_{i}-1} f(\tau, x(\tau), A x(\tau), B x(\tau)) \mathrm{d} \tau\right) \mathrm{d} H_{i}(s), t \in[0,1]$.

By $(I 1),(I 2)$ ' and Lemma 4 , we have $\left(Q_{1} x\right)(t)+\left(Q_{2} x\right)(t) \geq 0$ for all $t \in[0,1]$. For any $x \in \bar{B}_{R}$, by using $(B)$, we find

$$
\begin{aligned}
& |f(t, x(t), A x(t), B x(t))| \\
& \leq|f(t, x(t), A x(t), B x(t))-f(t, 0,0,0)|+|f(t, 0,0,0)| \\
& \leq a(t)|x(t)|+b(t)|A x(t)|+c(t)|B x(t)|+\tau_{0}, \forall t \in[0,1] .
\end{aligned}
$$

Then for any $x \in \bar{B}_{R}$ and all $t \in[0,1]$, we obtain by using the above inequality

$$
\begin{aligned}
\left|Q_{1} x(t)\right| & =\left|-\frac{1}{\Gamma(\alpha)} \int_{0}^{t}(t-s)^{\alpha-1} f(s, x(s), A x(s), B x(s)) \mathrm{d} s\right| \\
& \leq \frac{1}{\Gamma(\alpha)} \int_{0}^{t}(t-s)^{\alpha-1}\left(a(s)|x(s)|+b(s)|A x(s)|+c(s)|B x(s)|+\tau_{0}\right) \mathrm{d} s \\
& \leq \frac{\|x\|}{\Gamma(\alpha)} \int_{0}^{1}(1-s)^{\alpha-1}\left(a(s)+k_{0} b(s)+h_{0} c(s)\right) \mathrm{d} s+\frac{\tau_{0}}{\Gamma(\alpha)} \int_{0}^{t}(t-s)^{\alpha-1} \mathrm{~d} s \\
& \leq \omega_{2} R+\frac{t^{\alpha} \tau_{0}}{\Gamma(\alpha+1)} \leq \omega_{2} R+\frac{\tau_{0}}{\Gamma(\alpha+1)},
\end{aligned}
$$

because $(A x)(t) \leq \sup _{t \in[0,1]} x(t) \int_{0}^{t} K(t, s) \mathrm{d} s \leq k_{0}\|x\|$ and

$(B x)(t) \leq \sup _{t \in[0,1]} x(t) \int_{0}^{1} H(t, s) \mathrm{d} s \leq h_{0}\|x\|$ for all $t \in[0,1]$.

Therefore for any $z \in \bar{B}_{R}$ and all $t \in[0,1]$, we deduce by using (I4) that $\left|Q_{2} z(t)\right|$

$$
\begin{aligned}
\leq & \frac{t^{\alpha-1}}{\Delta \Gamma\left(\alpha-\beta_{0}\right)} \int_{0}^{1}(1-s)^{\alpha-\beta_{0}-1} f(s, z(s), A z(s), B z(s)) \mathrm{d} s \\
& +\frac{t^{\alpha-1}}{\Delta} \sum_{i=1}^{m} \frac{1}{\Gamma\left(\alpha-\beta_{i}\right)} \int_{0}^{1}\left(\int_{0}^{s}(s-\tau)^{\alpha-\beta_{i}-1} f(\tau, z(\tau), A z(\tau), B z(\tau)) \mathrm{d} \tau\right) \mathrm{d} H_{i}(s) \\
\leq & \frac{t^{\alpha-1}}{\Delta \Gamma\left(\alpha-\beta_{0}\right)} \int_{0}^{1}(1-s)^{\alpha-\beta_{0}-1} g(s) \mathrm{d} s \\
& +\frac{t^{\alpha-1}}{\Delta} \sum_{i=1}^{m} \frac{1}{\Gamma\left(\alpha-\beta_{i}\right)} \int_{0}^{1}\left(\int_{0}^{s}(s-\tau)^{\alpha-\beta_{i}-1} g(\tau) \mathrm{d} \tau\right) \mathrm{d} H_{i}(s) \\
\leq & \frac{1}{\Delta \Gamma\left(\alpha-\beta_{0}\right)} \int_{0}^{1} g(s) \mathrm{d} s+\frac{1}{\Delta} \sum_{i=1}^{m} \frac{1}{\Gamma\left(\alpha-\beta_{i}\right)} \int_{0}^{1}\left(\int_{0}^{1} g(\tau) \mathrm{d} \tau\right) \mathrm{d} H_{i}(s) \\
= & \|g\|_{L^{1}}\left(\frac{1}{\Delta \Gamma\left(\alpha-\beta_{0}\right)}+\frac{1}{\Delta} \sum_{i=1}^{m} \frac{1}{\Gamma\left(\alpha-\beta_{i}\right)}\left(H_{i}(1)-H_{i}(0)\right)\right) .
\end{aligned}
$$


Hence for $x, y \in \bar{B}_{R}$ and $t \in[0,1]$, we find

$$
\begin{aligned}
& \left|Q_{1} x(t)+Q_{2} y(t)\right| \\
& \leq\left|Q_{1} x(t)\right|+\left|Q_{2} y(t)\right| \leq \omega_{2} R+\frac{\tau_{0}}{\Gamma(\alpha+1)} \\
& \quad+\|g\|_{L^{1}}\left(\frac{1}{\Delta \Gamma\left(\alpha-\beta_{0}\right)}+\frac{1}{\Delta} \sum_{i=1}^{m} \frac{1}{\Gamma\left(\alpha-\beta_{i}\right)}\left(H_{i}(1)-H_{i}(0)\right)\right) \leq R .
\end{aligned}
$$

Therefore for $y_{1}, y_{2} \in \bar{B}_{R}$ and $t \in[0,1]$, by using (B) we obtain

$$
\begin{aligned}
&\left|Q_{2} y_{1}(t)-Q_{2} y_{2}(t)\right| \\
& \leq \frac{t^{\alpha-1}}{\Delta \Gamma\left(\alpha-\beta_{0}\right)} \int_{0}^{1}(1-s)^{\alpha-\beta_{0}-1} \mid f\left(s, y_{1}(s), A y_{1}(s), B y_{1}(s)\right) \\
&-f\left(s, y_{2}(s), A y_{2}(s), B y_{2}(s)\right) \mid \mathrm{d} s \\
&+\frac{t^{\alpha-1}}{\Delta} \sum_{i=1}^{m} \frac{1}{\Gamma\left(\alpha-\beta_{i}\right)} \int_{0}^{1}\left(\int_{0}^{s}(s-\tau)^{\alpha-\beta_{i}-1} \mid f\left(\tau, y_{1}(\tau), A y_{1}(\tau), B y_{1}(\tau)\right)\right. \\
&\left.-f\left(\tau, y_{2}(\tau), A y_{2}(\tau), B y_{2}(\tau)\right) \mid \mathrm{d} \tau\right) \mathrm{d} H_{i}(s) \\
& \leq \frac{1}{\Delta \Gamma\left(\alpha-\beta_{0}\right)} \int_{0}^{1}(1-s)^{\alpha-\beta_{0}-1}\left(a(s)\left|y_{1}(s)-y_{2}(s)\right|+b(s)\left|A y_{1}(s)-A y_{2}(s)\right|\right. \\
&\left.+c(s)\left|B y_{1}(s)-B y_{2}(s)\right|\right) \mathrm{d} s \\
&+\frac{1}{\Delta} \sum_{i=1}^{m} \frac{1}{\Gamma\left(\alpha-\beta_{i}\right)} \int_{0}^{1}\left(\int _ { 0 } ^ { s } ( s - \tau ) ^ { \alpha - \beta _ { i } - 1 } \left(a(\tau)\left|y_{1}(\tau)-y_{2}(\tau)\right|\right.\right. \\
&\left.\left.+b(\tau)\left|A y_{1}(\tau)-A y_{2}(\tau)\right|+c(\tau)\left|B y_{1}(\tau)-B y_{2}(\tau)\right|\right) \mathrm{d} \tau\right) \mathrm{d} H_{i}(s) \\
& \leq \frac{\left\|y_{1}-y_{2}\right\|}{\Delta \Gamma\left(\alpha-\beta_{0}\right)} \int_{0}^{1}(1-s)^{\alpha-\beta_{0}-1}\left(a(s)+k_{0} b(s)+h_{0} c(s)\right) \mathrm{d} s \\
&+\frac{\left\|y_{1}-y_{2}\right\|}{\Delta} \sum_{i=1}^{m} \frac{1}{\Gamma\left(\alpha-\beta_{i}\right)} \int_{0}^{1}\left(\int_{0}^{s}(s-\tau)^{\alpha-\beta_{i}-1}\left(a(\tau)+k_{0} b(\tau)+h_{0} c(\tau)\right) \mathrm{d} \tau\right) \mathrm{d} H_{i}(s) .
\end{aligned}
$$

So, we deduce

$$
\begin{aligned}
& \left|Q_{2} y_{1}(t)-Q_{2} y_{2}(t)\right| \\
& \leq \frac{\left\|y_{1}-y_{2}\right\|}{\Delta \Gamma\left(\alpha-\beta_{0}\right)} \int_{0}^{1}\left(a(s)+k_{0} b(s)+h_{0} c(s)\right) \mathrm{d} s \\
& \quad+\frac{\left\|y_{1}-y_{2}\right\|}{\Delta} \sum_{i=1}^{m} \frac{1}{\Gamma\left(\alpha-\beta_{i}\right)}\left(\int_{0}^{1}\left(a(\tau)+k_{0} b(\tau)+h_{0} c(\tau)\right) \mathrm{d} \tau\right)\left(H_{i}(1)-H_{i}(0)\right) \\
& =\left\|y_{1}-y_{2}\right\| \cdot\left\|a+k_{0} b+h_{0} c\right\|_{L^{1}}\left(\frac{1}{\Delta \Gamma\left(\alpha-\beta_{0}\right)}+\frac{1}{\Delta} \sum_{i=1}^{m} \frac{1}{\Gamma\left(\alpha-\beta_{i}\right)}\left(H_{i}(1)-H_{i}(0)\right)\right) \\
& =\omega_{3}\left\|y_{1}-y_{2}\right\|,
\end{aligned}
$$

where $\omega_{3}$ is given by (8). Because $\omega_{3}<1$, we conclude that $Q_{2}$ is a contraction mapping.

By using assumptions $(I 2)$ and (I5), we deduce that $Q_{1}$ is a continuous mapping. In addition, $Q_{1}$ is uniformly bounded on $\bar{B}_{R}$, because for any $x \in \bar{B}_{R}$, we find 


$$
\begin{aligned}
\left|Q_{1} x(t)\right| & =\left|\frac{1}{\Gamma(\alpha)} \int_{0}^{t}(t-s)^{\alpha-1} f(s, x(s), A x(s), B x(s)) \mathrm{d} s\right| \\
& \leq \frac{1}{\Gamma(\alpha)} \int_{0}^{t}(t-s)^{\alpha-1} g(s) \mathrm{d} s \leq \frac{1}{\Gamma(\alpha)} \int_{0}^{1} g(s) \mathrm{d} s \\
& =\frac{1}{\Gamma(\alpha)}\|g\|_{L^{1}}, \forall t \in[0,1],
\end{aligned}
$$

and then $\left\|Q_{1} x\right\| \leq \frac{1}{\Gamma(\alpha)}\|g\|_{L^{1}}$ for all $x \in \bar{B}_{R}$.

The operator $Q_{1}$ is also equicontinuous on $\bar{B}_{R}$. Indeed, let $x \in \bar{B}_{R}$, $t_{1}, t_{2} \in[0,1]$, with $t_{1}<t_{2}$. We have

$$
\begin{aligned}
\left|Q_{1} x\left(t_{2}\right)-Q_{1} x\left(t_{1}\right)\right| & \mid \frac{1}{\Gamma(\alpha)} \int_{0}^{t_{2}}\left(t_{2}-s\right)^{\alpha-1} f(s, x(s), A x(s), B x(s)) \mathrm{d} s \\
& -\frac{1}{\Gamma(\alpha)} \int_{0}^{t_{1}}\left(t_{1}-s\right)^{\alpha-1} f(s, x(s), A x(s), B x(s)) \mathrm{d} s \mid \\
\leq & \frac{1}{\Gamma(\alpha)} \int_{0}^{t_{1}}\left[\left(t_{2}-s\right)^{\alpha-1}-\left(t_{1}-s\right)^{\alpha-1}\right] f(s, x(s), A x(s), B x(s)) \mathrm{d} s \\
& +\frac{1}{\Gamma(\alpha)} \int_{t_{1}}^{t_{2}}\left(t_{2}-s\right)^{\alpha-1} f(s, x(s), A x(s), B x(s)) \mathrm{d} s \\
\leq & \phi_{0}\left\{\frac{1}{\Gamma(\alpha)} \int_{0}^{t_{1}}\left[\left(t_{2}-s\right)^{\alpha-1}-\left(t_{1}-s\right)^{\alpha-1}\right] \mathrm{d} s+\frac{1}{\Gamma(\alpha)} \int_{t_{1}}^{t_{2}}\left(t_{2}-s\right)^{\alpha-1} \mathrm{~d} s\right\} \\
= & \frac{\phi_{0}}{\Gamma(\alpha+1)}\left(t_{2}^{\alpha}-t_{1}^{\alpha}\right) \leq \frac{\phi_{0}\left(t_{2}-t_{1}\right)}{\Gamma(\alpha)},
\end{aligned}
$$

where $\phi_{0}=\sup \left\{f(t, x, y, z), t \in[0,1],|x| \leq R,|y| \leq k_{0} R,|z| \leq h_{0} R\right\}$. Then we obtain that $\left|Q_{1} x\left(t_{2}\right)-Q_{1} x\left(t_{1}\right)\right| \rightarrow 0$ as $t_{2}-t_{1} \rightarrow 0$.

By using the Arzela-Ascoli theorem, we deduce that $Q_{1}\left(\bar{B}_{R}\right)$ is relatively compact. By Theorem 2, we conclude that operator $Q_{1}+Q_{2}$ has at least one fixed point, and so problem $(E)-(B C)$ has at least one nonnegative solution.

\section{An Example}

Let $\alpha=10 / 3 \quad(n=4), \beta_{0}=11 / 5, \quad m=2, \quad \beta_{1}=1 / 2, \quad \beta_{2}=5 / 4, H_{1}(t)=t^{2}$ for all $t \in[0,1], H_{2}(t)=\{0$, for $t \in[0,1 / 2) ; 1$, for $t \in[1 / 2,1]\}$.

We consider the fractional differential equation

$$
D_{0+}^{10 / 3} x(t)+f(t, x(t), A x(t), B x(t))=0, t \in(0,1),
$$

with the boundary conditions

$$
\left(B C_{0}\right) \quad x(0)=x^{\prime}(0)=x^{\prime \prime}(0)=0, D_{0+}^{11 / 5} x(1)=2 \int_{0}^{1} t D_{0+}^{1 / 2} x(t) \mathrm{d} t+D_{0+}^{5 / 4} x\left(\frac{1}{2}\right),
$$

where $A x(t)=\int_{0}^{t} K(t, s) x(s) \mathrm{d} s$ and $B x(t)=\int_{0}^{1} H(t, s) x(s) \mathrm{d} s$ for all $t \in[0,1]$, with $K(t, s)=\mathrm{e}^{-t} s^{2}$ for all $t, s \in[0,1]$ with $s \leq t$, and 
$H(t, s)=\mathrm{e}^{-2 t}\left(s^{3}+1\right)$ for all $t, s \in[0,1]$. Then we obtain $\Delta \approx 0.85599748>0$ and $\sigma \approx 1.24445843$. So assumptions (Il) and (I5) are satisfied.

We define the function

$$
f(t, x, y, z)=\frac{t+1}{2}+\frac{\mathrm{e}^{-t} x}{(1+k)(1+x)}+\frac{\mathrm{e}^{-2 t} y}{\left(1+k^{2}\right)(1+y)}+\frac{\mathrm{e}^{-3 t} z}{\left(1+k^{3}\right)(1+z)},
$$

for all $t \in[0,1]$ and $x, y, z \in \mathbb{R}_{+}$with $k \geq 1$. We deduce that $k_{0}=\frac{1}{3 \mathrm{e}}$ and $h_{0}=\frac{5}{4}$. Besides we obtain the inequalities

$$
|f(t, x, y, z)-f(t, \bar{x}, \bar{y}, \bar{z})| \leq \frac{\mathrm{e}^{-t}}{1+k}|x-\bar{x}|+\frac{\mathrm{e}^{-2 t}}{1+k^{2}}|y-\bar{y}|+\frac{\mathrm{e}^{-3 t}}{1+k^{3}}|z-\bar{z}|,
$$

for all $t \in[0,1], x, y, z, \bar{x}, \bar{y}, \bar{z} \in \mathbb{R}_{+}$, and

$$
|f(t, x, y, z)| \leq \frac{t+1}{2}+\frac{\mathrm{e}^{-t}}{1+k}+\frac{\mathrm{e}^{-2 t}}{1+k^{2}}+\frac{\mathrm{e}^{-3 t}}{1+k^{3}}, \forall t \in[0,1], x, y, z \in \mathbb{R}_{+} .
$$

We define $a(t)=\frac{\mathrm{e}^{-t}}{1+k}, \quad b(t)=\frac{\mathrm{e}^{-2 t}}{1+k^{2}}, c(t)=\frac{\mathrm{e}^{-3 t}}{1+k^{3}}$, and $g(t)=\frac{t+1}{2}+\frac{\mathrm{e}^{-t}}{1+k}+\frac{\mathrm{e}^{-2 t}}{1+k^{2}}+\frac{\mathrm{e}^{-3 t}}{1+k^{3}}$, for all $t \in[0,1]$. We have $a, b, c \in L^{1}(0,1)$ and $g \in L^{1}(0,1)$. So assumptions (I2), (I), (I4) are also satisfied.

In addition, we find $\omega_{1}=\frac{\mathrm{e}-1}{\mathrm{e}(1+k)}+\frac{\mathrm{e}^{2}-1}{6 \mathrm{e}^{3}\left(1+k^{2}\right)}+\frac{5\left(\mathrm{e}^{3}-1\right)}{12 \mathrm{e}^{3}\left(1+k^{3}\right)} \leq \frac{\mathrm{e}-1}{2 \mathrm{e}}+\frac{\mathrm{e}^{2}-1}{12 \mathrm{e}^{3}}+\frac{5\left(\mathrm{e}^{3}-1\right)}{24 \mathrm{e}^{3}} \approx 0.540529$, and so $\omega_{1}<1 / \sigma \approx 0.8035624$. Therefore, by Theorem 3, we conclude that problem $\left(\mathrm{E}_{0}\right)-\left(\mathrm{BC}_{0}\right)$ has at least one nonnegative and nontrivial solution.

\section{Conclusion}

In this paper, we investigated the existence of nonnegative solutions for the Riemann-Liouville fractional differential equation with integral terms (E) supplemented with the boundary conditions (BC) which contain Riemann-Liouville fractional derivatives of different orders and Riemann-Stieltjes integrals, by using the Banach contraction mapping principle and the Krasnosel'skii fixed point theorem for the sum of two operators. For some future research directions, we have in mind the study of the existence, nonexistence and multiplicity of solutions or positive solutions for fractional differential equations subject to other boundary conditions.

\section{Acknowledgements}

The authors thank the referee for his/her valuable comments and suggestions.

\section{Conflicts of Interest}

The authors declare no conflicts of interest regarding the publication of this paper. 


\section{References}

[1] Henderson, J. and Luca, R. (2017) Existence of Nonnegative Solutions for a Fractional Integro-Differential Equation. Results in Mathematics, 72, 747-763. https://doi.org/10.1007/s00025-017-0655-y

[2] Wang, Y. and Liu, L. (2017) Uniqueness and Existence of Positive Solutions for the Fractional Integro-Differential Equation. Boundary Value Problems, 2017, Article No. 12. https://doi.org/10.1186/s13661-016-0741-1

[3] Agarwal, R.P. and Luca, R. (2019) Positive Solutions for a Semipositone Singular Riemann-Liouville Fractional Differential Problem. International Journal of Nonlinear Sciences and Numerical Simulation. (In Press) https://doi.org/10.1515/ijnsns-2018-0376

[4] Henderson, J. and Luca, R. (2015) Boundary Value Problems for Systems of Differential, Difference and Fractional Equations: Positive Solutions. Elsevier, Amsterdam.

[5] Zhou, Y. (2014) Basic Theory of Fractional Differential Equations. World Scientific, Singapore. https://doi.org/10.1142/9069

[6] Ahmad, B. and Luca, R. (2018) Existence of Solutions for a System of Fractional Differential Equations with Coupled Nonlocal Boundary Conditions. Fractional Calculus and Applied Analysis, 21, 423-441. https://doi.org/10.1515/fca-2018-0024

[7] Ahmad, B. and Ntouyas, S.K. (2015) Existence Results for a Coupled System of Caputo Type Sequential Fractional Differential Equations with Nonlocal Integral Boundary Conditions. Applied Mathematics and Computation, 266, 615-622. https://doi.org/10.1016/j.amc.2015.05.116

[8] Aljoudi, S., Ahmad, B., Nieto, J.J. and Alsaedi, A. (2016) A Coupled System of Hadamard Type Sequential Fractional Differential Equations with Coupled Strip Conditions. Chaos Solitons Fractals, 91, 39-46. https://doi.org/10.1016/j.chaos.2016.05.005

[9] Guo, L., Liu, L. and Wu, Y. (2018) Iterative Unique Positive Solutions for Singular p-Laplacian Fractional Differential Equation System with Several Parameters. Nonlinear Analysis: Modelling and Control, 23, 182-203. https://doi.org/10.15388/NA.2018.2.3

[10] Henderson, J., Luca, R. and Tudorache, A. (2015) On a System of Fractional Differential Equations with Coupled Integral Boundary Conditions. Fractional Calculus and Applied Analysis, 18, 361-386. https://doi.org/10.1515/fca-2015-0024

[11] Liu, L., Li, H., Liu, C. and Wu, Y. (2017) Existence and Uniqueness of Positive Solutions for Singular Fractional Differential Systems with Coupled Integral Boundary Value Problems. Journal of Nonlinear Sciences and Applications, 10, 243-262. https://doi.org/10.22436/jnsa.010.01.24

[12] Shen, C., Zhou, H. and Yang, L. (2016) Positive Solution of a System of Integral Equations with Applications to Boundary Value Problems of Differential Equations. Advances in Difference Equations, 2016, Article No. 260. https://doi.org/10.1186/s13662-016-0953-9

[13] Xu, J. and Wei, Z. (2016) Positive Solutions for a Class of Fractional Boundary Value Problems. Nonlinear Analysis. Modelling and Control, 21, 1-17. https://doi.org/10.15388/NA.2016.1.1

[14] Zhang, X. and Zhong, Q. (2018) Triple Positive Solutions for Nonlocal Fractional Differential Equations with Singularities Both on Time and Space Variables. Applied Mathematics Letters, 80, 12-19. https://doi.org/10.1016/j.aml.2017.12.022 
[15] Baleanu, D., Diethelm, K., Scalas, E. and Trujillo, J.J. (2012) Fractional Calculus Models and Numerical Methods. Series on Complexity, Nonlinearity and Chaos. World Scientific, Boston. https://doi.org/10.1142/9789814355216

[16] Das, S. (2008) Functional Fractional Calculus for System Identification and Control. Springer, New York.

[17] Kilbas, A.A., Srivastava, H.M. and Trujillo, J.J. (2006) Theory and Applications of Fractional Differential Equations. North-Holland Mathematics Studies, 204, Elsevier Science B.V., Amsterdam.

[18] Klafter, J., Lim, S.C. and Metzler, R. (2011) Fractional Dynamics in Physics. World Scientific, Singapore. https://doi.org/10.1142/8087

[19] Podlubny, I. (1999) Fractional Differential Equations. Academic Press, San Diego.

[20] Sabatier, J., Agrawal, O.P. and Machado, J.A.T. (2007) Advances in Fractional Calculus: Theoretical Developments and Applications in Physics and Engineering. Springer, Dordrecht. https://doi.org/10.1007/978-1-4020-6042-7

[21] Samko, S.G., Kilbas, A.A. and Marichev, O.I. (1993) Fractional Integrals and Derivatives. Theory and Applications. Gordon and Breach, Yverdon.

[22] Arafa, A.A.M., Rida, S.Z. and Khalil, M. (2012) Fractional Modeling Dynamics of $\mathrm{HIV}$ and $\mathrm{CD}^{+} \mathrm{T}$-Cells during Primary Infection. Nonlinear Biomedical Physics, 6 , 1-7. https://doi.org/10.1186/1753-4631-6-1

[23] Ding, Y. and Ye, H. (2009) A Fractional-Order Differential Equation Model of HIV Infection of CD4 ${ }^{+}$T-Cells. Mathematical and Computer Modelling, 50, 386-392. https://doi.org/10.1016/j.mcm.2009.04.019

[24] Djordjevic, V., Jaric, J., Fabry, B., Fredberg, J. and Stamenovic, D. (2003) Fractional Derivatives Embody Essential Features of Cell Rheological Behavior. Annals of Biomedical Engineering, 31, 692-699. https://doi.org/10.1114/1.1574026

[25] Ge, Z.M. and Ou, C.Y. (2008) Chaos Synchronization of Fractional Order Modified Duffing Systems with Parameters Excited by a Chaotic Signal. Chaos Solitons Fractals, 35, 705-717. https://doi.org/10.1016/j.chaos.2006.05.101

[26] Luca, R. and Tudorache, A. (2014) Positive Solutions to a System of Semipositone Fractional Boundary Value Problems. Advances in Difference Equations, 2014, Article No. 179. https://doi.org/10.1186/1687-1847-2014-179

[27] Metzler, R. and Klafter, J. (2000) The Random Walks Guide to Anomalous Diffusion: A Fractional Dynamics Approach. Physics Reports, 339, 1-77. https://doi.org/10.1016/S0370-1573(00)00070-3

[28] Sokolov, I.M., Klafter, J. and Blumen, A. (2002) A Fractional Kinetics. Physics Today, 55, 48-54. https://doi.org/10.1063/1.1535007

[29] Henderson, J. and Luca, R. (2017) Existence of Positive Solutions for a Singular Fractional Boundary Value Problem. Nonlinear Analysis: Modelling and Control, 22, 99-114. https://doi.org/10.15388/NA.2017.1.7

[30] Deimling, K. (1985) Nonlinear Functional Analysis. Springer-Verlag, Berlin, Heidelberg, New York, Tokyo.

[31] Krasnosel'skii, M.A. (1955) Two Remarks on the Method of Successive Approximations. Uspekhi Matematicheskikh Nauk, 10, 123-127. 